\title{
Impacto del Entrenamiento del Balance a través de Realidad Virtual en una Población de Adultos Mayores
}

\author{
Impact of Balance Training with a Virtual Reality in Elderly
}

\begin{abstract}
"Valeska Gatica Rojas; ${ }^{* *}$ Edith Elgueta Cancino; ${ }^{* * *}$ Cristian Vidal Silva; ${ }^{* * * * *}$ Mario Cantin López \& ${ }^{* * * * * *}$ Jorge Fuentealba Arcos
\end{abstract}
GATICA, R. V.; ELGUETA, C. E.; VIDAL, S. C.; CANTIN, L. M. \& FUENTEALBA, A. J. Impacto del entrenamiento del balance a través de realidad virtual en una población de adultos mayores. Int. J. Morphol., 28(1):303-308, 2010.

RESUMEN: La tercera edad tanto en Chile como en el mundo es una población creciente, que se caracteriza por que sus habilidades motoras sufren un deterioro progresivo y natural, destaca la pérdida del balance como una más afectadas; facilitando las caídas y por consiguiente lesiones traumáticas graves, como es la fractura de cadera. Este trabajo persigue reeducar el balance y control postural en los adultos mayores a través del uso de realidad virtual. El estudio se llevo a cabo en el Laboratorio de Biomecánica de la Universidad de Talca. Participaron 20 sujetos adultos mayores (edad promedio 69 años). Se utilizo un modelo de intervención de 8 semanas con una frecuencia de 3 veces a la semana, 20 minutos cada sesión. Se evaluó el balance y control postural a partir de una plataforma posturográfica estática (Estandar y Tándem) antes de comenzar la intervención, a las 3, 6 y 8 semanas de tratamiento. El área y velocidad promedio disminuyeron de manera significativa en la fase de vista al frente en la prueba de apoyo Bipodal o Estandar lo que representa una disminución del $30 \%$ respecto de los valores iniciales. La velocidad promedio se redujo un $4 \%$ respecto al inicio (p<0,01). En la prueba Tándem, él área de desplazamiento del centro de presión (COP) disminuyo de manera significativa en la fase de vista al frente, lo que significa una disminución progresiva del 28\% ( $<<0,01)$. El entrenamiento con RV (Nintendo Wii Fit) contribuye de manera significativa en mejorar el balance y el control postural en los adultos mayores. Aspectos observados en la variables posturograficas tales como: área de desplazamiento del COP en vista al frente para la prueba Estándar y Tándem, en la velocidad promedio de excursión del COP para la fase de vista al frente en la prueba Estandar y ojos cerrados para Tándem.

PALABRAS CLAVE: Balance; centro de presión; Adulos mayores; Realidad virtual.

\section{INTRODUCCIÓN}

La alteración en el sistema de control postural en edad avanzada, se ha asociado a la degeneración progresiva de los sistemas responsables de la postura, entre ellos los sistemas somatosensorial (propioceptores y mecanorreceptores), visual y vestibular (van Asten et al., 1988a). Este tipo de alteración es una de las principales causas que llevan a la pérdida del balance, facilitando las caídas en la población con una importante prevalencia en los adultos mayores (van Asten et al., 1988b).

Por otro parte, la degeneración del balance en el adulto mayor (AM) ha estimulado la investigación en los últimos años sobre cómo interactúan los sistemas de control postural y cómo cuantificarlos en cualquier etapa de la vida
(Gage et al., 2004). Los estudios muestran que el balance se pierde cuando el desplazamiento del centro de presión (COP) cae fuera de los límites de estabilidad, es decir, fuera de la óptima posición del COP dentro de la base de sustentación. Se ha demostrado que el envejecimiento está asociado con un incremento del desplazamiento del COP (afectando las variables de área y velocidad promedio) durante la posición de pie (Onambele et al., 2006).

Diversos autores señalan que el sistema más solicitado por el AM para controlar la postura es la visión (Gage et al., 2007), y cualquier alteración de éste puede generar repercusiones negativas sobre el balance postural facilitando las caídas (Redfern et al., 2009). La retroalimentación visual juega

* Facultad de Ciencias de la Salud, Departamento de Ciencias Básicas, Escuela de Kinesiología. Universidad de Talca, Chile.

** Licenciada en Kinesiología. Facultad de Ciencias de la Salud, Laboratorio de Biomecánica, Escuela de Kinesiología. Universidad de Talca, Chile.

*** Magister en Ingeniería en Informática. Escuela de Ingeniería Informática Empresarial. Universidad de Talca, Chile.

**** Departamento de Ciencias Básicas. Unidad de Anatomía Normal. Universidad de Talca, Chile.

${ }^{* * * * * *}$ Facultad de Ciencias Biológicas. Departamento de Fisiología. Universidad de Concepción, Chile. 
un papel importante en la postura. Muchos investigadores han encontrado que la única contribución del sistema visual es en el balance (Wolsley et al., 1996). En particular, los movimientos detectados por la retina pueden ser usados para determinar los movimientos de sí mismo o los movimientos del ambiente. Sin embargo, algunas veces, existe una ambigüedad entre la sensación y la dificultad para establecer las diferencias entre los movimientos de sí mismo y los ambientales. Como resultado de estos, puede ocurrir una inestabilidad.

Además de la visión, el balance postural también puede ser influenciado por la información propioceptiva. La propiocepción es una variación especializada de la modalidad sensorial y abarca la sensaciones del movimiento articular (kinestesia) y posición articular (sentido de reposición articular). Contribuye a la programación motora y a los reflejos musculares para la estabilidad articular dinámica (Lee \& Lin, 2008). La propiocepción desde los tobillos y la planta de los pies contribuye de manera significativa sobre el balance. Cuando ésta información propioceptiva está disponible, la estabilidad postural aumenta. Sin embargo, cuando la propiocepción es reducida, como se observa en los casos de neuropatía periférica, el control postural vuelve a disminuir. En pacientes con una reducida sensación propioceptiva, es común encontrar una gran preponderancia a solicitar los sistemas visuales y vestibulares para mantener el balance.

Respecto al sistema vestibular, es interesante saber que las señales vestibulares por sí solas no pueden entregar al sistema nervioso central (SNC) una imagen verdadera de cómo el cuerpo se mueve en el espacio. Por lo tanto, la información de los receptores sensoriales del aparato vestibular interactúan con la información del sistema visual y somatosensorial para producir una alineación adecuada del cuerpo y control postural (Diener \& Dichgans, 1988). Existe evidencia de que pacientes con una reducida acción vestibular, tienden a utilizar masivamente las claves visuales (Peterka, 2002) y propioceptivas para mantener el balance.

Las mejoras en el control postural son comúnmente alcanzadas a través de programas de entrenamiento del balance, los cuales han mostrado ser efectivos en sujetos saludables y lesionados (Rothermel et al., 2004). La herramienta de entrenamiento para la rehabilitación del balance ha sido por excelencia con el Plato de Freeman o Wobble board, que es comúnmente usado en la rehabilitación de tobillos funcionalmente inestables. En este sentido, se ha demostrado que entrenar la inestabilidad de tobillo con Wobble board con una frecuencia de 3 veces por semana durante 4 semanas, mejora la habilidad de pararse en una pierna y la oscilación postural en participantes con tobillo funcionalmente inestable (Clark \& Burden, 2005; Wester et al., 1996). Lo interesante de este entrenamiento es el protocolo propuesto, el cual está enfocado a solicitar los tres planos de movimiento (sagital, frontal y transversal). Por otra parte, se ha observado que entrenar el balance por medio de ambientes virtuales disminuye de manera significativa el desplazamiento del COP, en sujetos jóvenes con trastornos neuromotores (Betker et al., 2006). En este sentido, se ha evidenciado una gran contribución de la realidad virtual (RV) hacia la medicina y rehabilitación. Definir RV es difícil; sin embargo, se entiende como una simulación por computadora, dinámica y tridimensional, con alto contenido gráfico, acústico y táctil, orientada a la visualización de situaciones complejas, resultando el sujeto inmerso en ambientes altamente participativos, de origen artificial, pero con un gran porcentaje de realismo (Parra et al., 2001).

Considerando que nuestra muestra de estudio no son sujetos jóvenes como los planteados en la literatura, sino que son adultos mayores donde el balance pudiese estar bastante deteriorado; y las posibilidades de restablecerlo por medio del Wobble board involucra un riesgo mayor que en sujetos jóvenes. Por lo tanto, hemos escogido ambientes virtuales, los cuales son considerados como interesantes, lúdicos y por sobre todo muy seguros. Nuestro objetivo de investigación fue reeducar el balance y control postural en los adultos mayores, a través del uso de realidad virtual, inducida con una plataforma informática diseñada como un juego de entretención.

\section{MATERIAL Y MÉTODO}

Estudio experimental e intervencional. La muestra fue conformada por 20 sujetos adultos mayores (edad promedio 69 años). Todos informados del estudio, dieron su consentimiento escrito. Se utilizó un modelo de intervención de 8 semanas con una frecuencia de 3 veces a la semana, 20 minutos cada sesión. El protocolo de entrenamiento fue basado en el propuesto por Clark \& Burden, adaptado desde Wester et al. En este sentido, hemos planteado un entrenamiento seguro, entretenido y que garantiza trabajar los tres planos de movimientos (sagital, frontal y transverso). Por medio del equipo de Wii Fit y su periférico Balance Board se ejecutó el protocolo descrito en la Tabla I.

Se evaluó el balance y control postural a partir de una plataforma posturográfica estática antes de comenzar la intervención, a las 3, 6 y 8 semanas de tratamiento. La evaluación posturográfica consistió en una prueba de apoyo bipodal (Estandar) y de apoyo bipodal con pies en línea (Tándem). Las pruebas fueron completadas con dos fases: vista al frente y ojos cerrados; las cuales permitieron aislar la participación del sistema visual en la evaluación del control postural. Las variables posturografica analizadas fueron: área de desplazamiento del COP, velocidad promedio de desplazamiento del 
Tabla I. Protocolo Wii Fit. Entrenamiento del balance a partir de los 3 planos de movimiento (Modificado desde Wester et al., 1996 y Clark \& Burden, 2005).

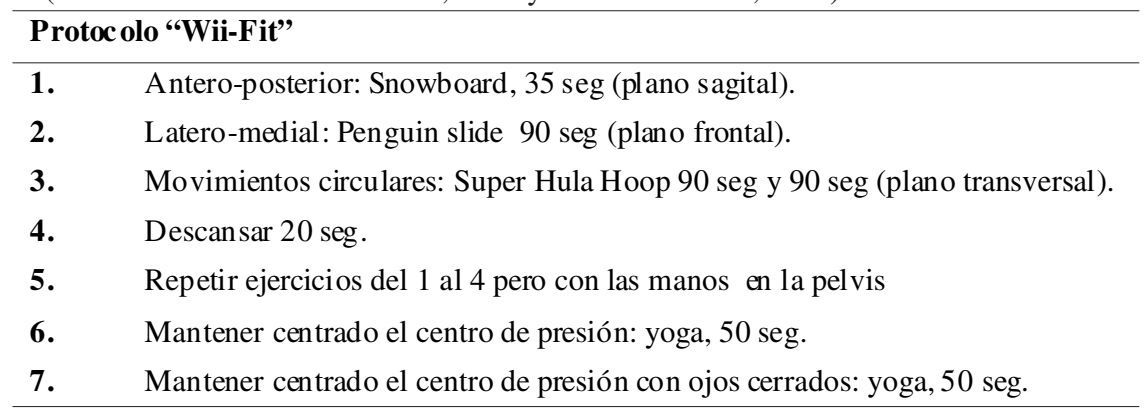

COP y las bandas de energía de la señal posturográfica $4 \mathrm{~Hz}$, $2 \mathrm{~Hz}, 1 \mathrm{~Hz}, 1 / 2 \mathrm{~Hz}, 1 / 4 \mathrm{~Hz}, 1 / 8 \mathrm{~Hz}$ y $1 / 16 \mathrm{~Hz}$.

Las pruebas de significancia estadística se realizaron utilizando la prueba test " $t$ " de Student emparejada, un nivel de probabilidad $\mathrm{p}<0,05, \mathrm{p}<0,01 \mathrm{y} \mathrm{p}<0,001$ se considero estadísticamente significativo (X; SEM).

\section{RESULTADOS}

Evaluación Posturográfica Estandar. El área y velocidad promedio disminuyeron de manera significativa en la fase de vista al frente en la prueba de apoyo Bipodal o Estandar, valores observados para el área fueron: para la semana 3 $(0,01026 \pm 0,001)$, para la semana $6(0,00784 \pm 0,001)$ y para la semana $8(0,0074 \pm 0,001)$ lo que representa una disminución del 28\% respecto de los valores iniciales (Fig. 1). Estos datos son congruentes con la banda de energía 1/16 (Redfern et al., 2001), la cual se relaciona con la utilización del sistema visual por parte de los AM para el manejo del control postural. La banda $1 / 8$ y $1 / 2$ relacionadas con el sistema vestibular (Redfern et al., 2001) presentan mejoras significativas.
La velocidad promedio en la semana $3(0,23476 \pm 0,004)$ y en la semana $8(0,22665 \pm 0,002)$, presentaron una reducción del $4 \%$ respecto al inicio (p<0,01) (Fig. 2).

Evaluación Posturográfica Tándem. En la prueba Tándem, él área de desplazamiento del COP disminuyo de manera significativa en la fase de vista al frente, observándose en la semana $3(0,03714 \pm 0,007), 6(0,02853 \pm 0,004)$ y 8 $(0,02689 \pm 0,004)$, lo que significa una disminución progresiva del 28\% (p<0,01) (Fig. 3).

De la misma forma, la velocidad promedio evidencio una disminución significativa en la fase de ojos cerrados (semana 3 X: 0,50136 $\pm 0,02$; semana 6 X: 0,46545 $\pm 0,02$ y semana 8 X: 0,45201 $\pm 0,02)(\mathrm{p}<0,01)$ (Fig. 4). La banda de energía $1 / 16$ la cual se relaciona con la utilización del sistema visual por parte de los AM disminuye significativamente en la fase de vista al frente $\mathrm{p}<0,01$.

El sistema vestibular realiza un aporte significativo en la fase de vista al frente, observado en las bandas $1 / 8$ y $1 /$ 4 las cuales se relacionan con el utrículo y sáculo.

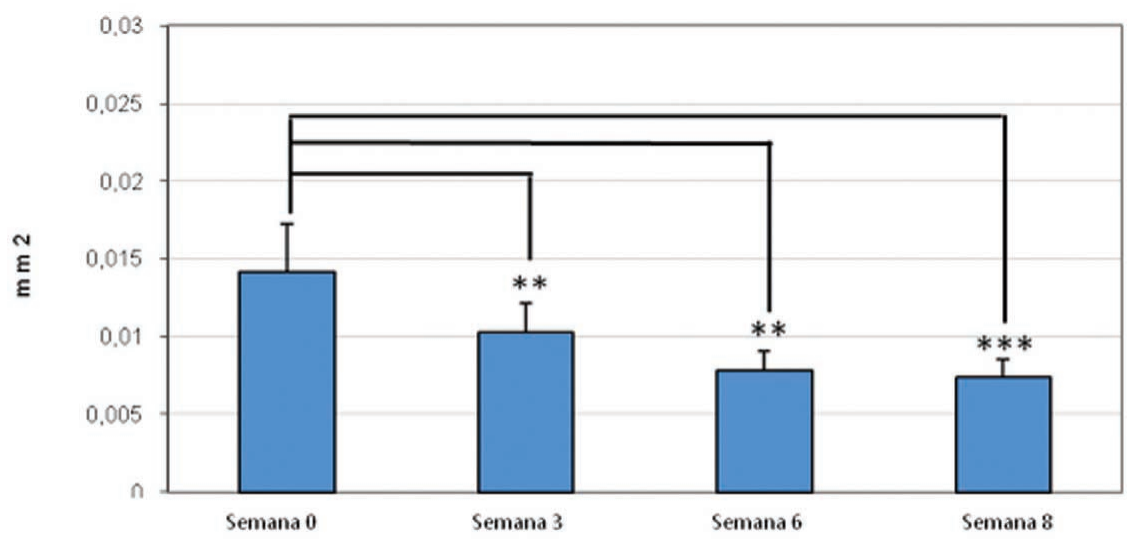

Evaluaciones
Fig. 1. Área en fase de vista al frente. Posturografía Estándar. Diferencias significativas a las 3, 6 y 8 semanas de entrenamiento con Wii Fit. $\mathrm{p}<0,01(* *)$ y $\mathrm{p}<0,001$ $(* * *)$. 


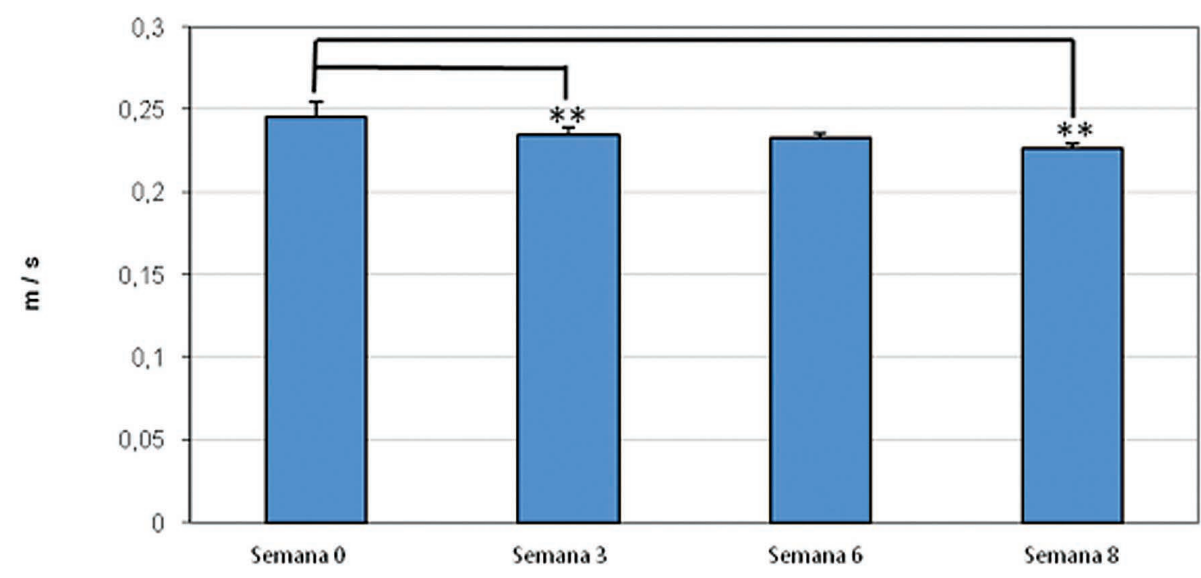

Evaluaciones

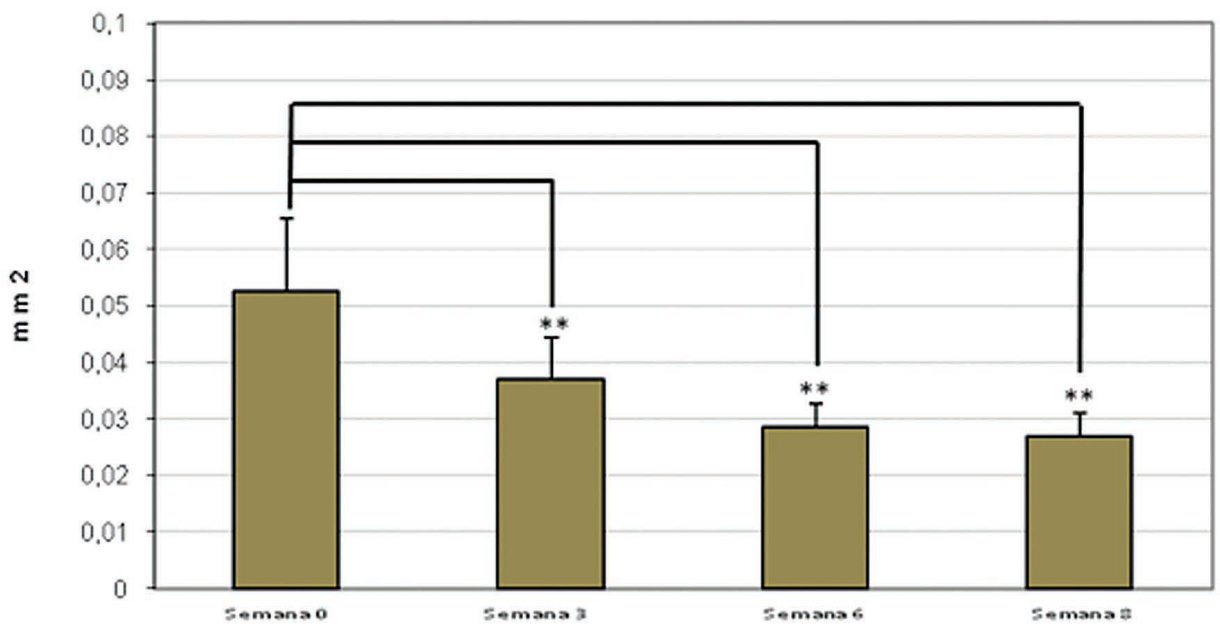

Evaluaciones

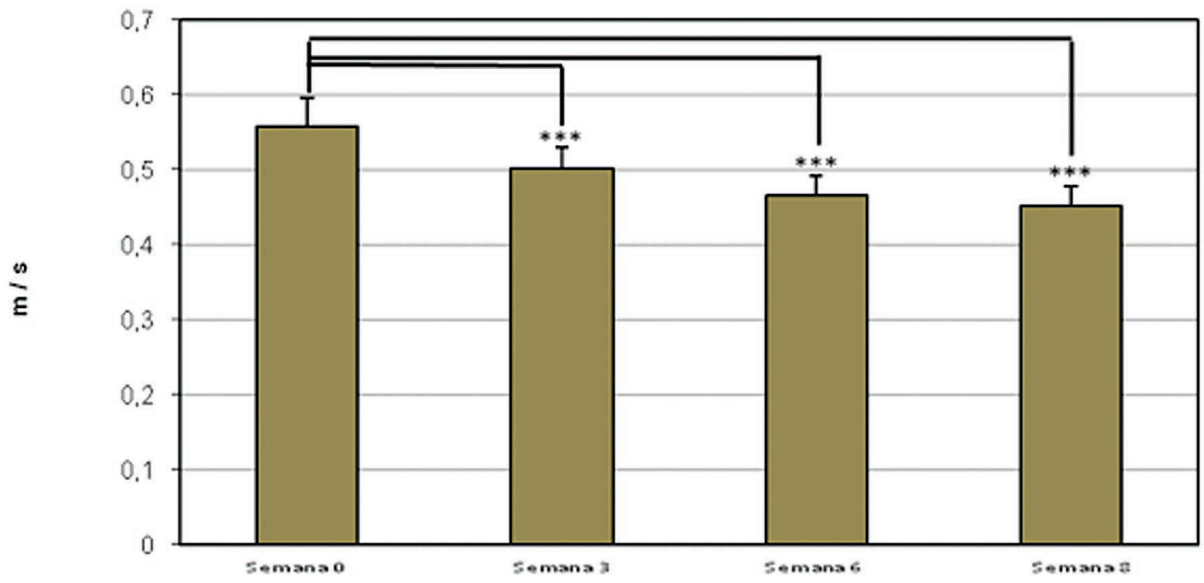

Evaluaciones
Fig. 2. Velocidad en la fase de vista al frente. Posturografía Estándar (Evaluaciones n=20). Diferencias significativas $\mathrm{p}<0,01$ (**) y $\mathrm{p}<0,001(* * *)$.

Fig. 3. Área de desplazamiento del COP en la fase de vista al frente. Posturografia Tánde. Diferencias significativas $\mathrm{p}<0,01(* *)$.

Fig. 4. Velocidad promedio en la fase de ojos cerrados. Posturografia Tándem. Diferencias significativas $\mathrm{p}<0,001(* * *)$. 


\section{DISCUSIÓN}

Los resultados presentados confirman que efectivamente el entrenamiento programado para este tipo de pacientes mostró cambios sustantivos en las variables de velocidad media y área de desplazamiento del COP en los adultos mayores en las modalidades posturograficas Estándar y Tándem.

En este sentido, al ser un entrenamiento del balance era esperable que estas variables disminuyeran de manera significativa, puesto que esto indicaría una mejora del control postural (Fujita et al., 2005; Onambele et al.; Piirtola \& Era, 2006; Seigle et al., 2009), especialmente en los adultos mayores; ya que se sabe, estos están más asociados a una disminución del control postural con un consiguiente aumento de la velocidad, área y otras variables como el desplazamiento del centro de presión.

La disminución significativa del área y velocidad media en la fase de vista al frente cuando se está con apoyo bipodal (Estándar); reafirma la enorme utilidad y apoyo en el sistema visual para controlar la postura por parte de los AM, pero también nos permite señalar que este programa de entrenamiento indujo una reeducación del balance postural, sugiriendo que los individuos utilizaron de forma más eficiente sus herramientas posturales disponibles. Esta mejora significativa en el balance esta potenciada por el sistema visual, como hemos dicho más eficiente, lo que queda representado por la disminución significativa de la banda de energía de la señal $1 / 16 \mathrm{~Hz}$, descrita como el referente de la utilización del sistema visual (Redfern et al., 2001). Además de la eficiencia obtenida en el sistema visual después del entrenamiento, también se evidencio una disminución en la bandas $1 / 8$ y1/2 Hz, que como se mencionó están relacionadas con el sistema vestibular, específicamente utrículo, sáculo y canales semicirculares respectivamente. Por lo tanto, los AM después del entrenamiento mejoran progresivamente la eficiencia del sistema visual y vestibular, parámetros que contribuyen de forma crucial con la reeducación del balance postural.
Por otra parte, las disminuciones en torno al área de desplazamiento del COP observadas en la prueba Tándem, dan cuenta nuevamente de la utilización del sistema visual para controlar la postura. Es importante señalar que la prueba Tándem realizada en la plataforma posturografica, es extrema, en el sentido en que ubica al cuerpo del individuo en un momento de gran inestabilidad. De manerasimilar que en la prueba Estandar, el sistema visual después de un periodo de entrenamiento con realidad virtual se torna más eficiente para controlar el balance, lo que se correlaciona con la disminución de la banda 1/16 después del entrenamiento. Además, el sistema vestibular realiza un gran aporte al balance tanto en la fase de vista al frente como en la fase de ojos cerrados. Durante esta prueba se observo una disminución de la velocidad promedio en la fase de ojos cerrados, siendo relevante en la génesis de un movimiento más controlado y de mejor calidad después del entrenamiento con RV.

Finalmente, podemos señalar que la inmersión en otra realidad, ficticia pero integradora podría explicar los beneficios en el balance sobre todo, en la disminución del área de desplazamiento del centro de presión en los adultos mayores. Algunos estudios señalan que no teniendo conciencia o no estando enfocado en mantener la postura correcta se obtendría un mejor control automático de la postura (Vuillerme \& Nafati, 2007).

Los resultados obtenidos en este estudio, apoyan la hipótesis de que el entrenamiento con realidad virtual (Wii Fit y Balance Board) mejora el balance y el control postural en sujetos adultos mayores, a partir de períodos de 3 semanas o superiores conformando de esta manera, una estrategia de salud útil para los AM.

\section{AGRADECIMIENTOS}

Este trabajo conto con la colaboración de la Facultad de Ciencias de la Salud y la Dirección de Investigación (DI) proyecto Investigadores Iniciales VAC 600499.

GATICA, R. V.; ELGUETA, C. E.; VIDAL, S. C.; CANTIN, L. M. \& FUENTEALBA, A. J. Impact of balance training with a virtual reality in elderly.Int. J. Morphol., 28(1):303-308, 2010.

SUMMARY: The elderly in Chile and in the world, is a growing population, characterized by their motor skills suffer a progressive and natural deterioration highlight the balance as a loss most affected by facilitating falls and therefore severe trauma injuries, such as hip fracture. This work seeks re-educate balance and postural control in older adults through the use of virtual reality. The study populations included 20 older individuals (mean $=69$ years). All participants gave written, informed consent to participate in the study. The subjects were training with a intervention model of tree times a week during 8 weeks with 20 minutes per session. Two test were applied: bipedal stance and Tándem stance, and was performed four times, before the intervention at 3,6 and 8 weeks of the training. In bipedal stance there were significant changes in the area and velocity of displacement of the center of pressure (DCoP) in open eyes phase. The area that represent about a $30 \%$ of the beginning values, and the velocity equivalent to $4 \%$ to the beginning values $(\mathrm{p}<0.01)$. In Tándem stance there were significant changes in the area of DCoP in open eyes phase with a decrease of $28 \%$ $(\mathrm{p}<0.01)$. The training with virtual reality (Nintendo Wii Fit) generate a significant decrease in older individuals in balance. Parameters like the area in open eyes phase in both test and the mean velocity in open eyes phase of bipedal stance test and in closed eyes for Tándem stance test. 


\section{REFERENCIAS BIBLIOGRÁFICAS}

Betker, A. L.; Szturm, T.; Moussavi, Z. K. \& Nett, C. Video game-based exercises for balance rehabilitation: a single-subject design. Arch. Phys. Med. Rehabili., 87(8):1141-9, 2006.

Clark, V. \& Burden, A. A 4-week wobble board exercise programme improved muscle onset latency and perceived stability in individuals with a functionally unstable ankle. Phys. Ther. Sport, 6:181-7, 2005.

Diener, H. C. \& Dichgans, J. On the role of vestibular, visual and somatosensory information for dynamic postural control in humans. Prog. Brain Res., 76:25362, 1988.

Fujita, T.; Nakamura, S.; Ohue, M.; Fujii, Y.; Miyauchi, A.; Takagi, Y. \& Tsugeno, H. Effect of age on body sway assessed by computerized posturography. J. Bone Miner. Metab., 23(2):152-6, 2005.

Gage, W. H.; Frank, J. S.; Prentice, S. D. \& Stevenson, P. Organization of postural responses following a rotational support surface perturbation, after TKA: Sagittal plane rotations. Gait Posture, 25(1):112-20, 2007.

Gage, W. H.; Winter, D. A.; Frank, J. S. \& Adkin, A. L. Kinematic and kinetic validity of the inverted pendulum model in quiet standing. Gait Posture, 19(2):124-32, 2004.

Lee, A. J. \& Lin, W. H. Twelve-week biomechanical ankle platform system training on postural stability and ankle proprioception in subjects with unilateral functional ankle instability. Clin. Biomech. (Bristol, Avon), 23(8):1065-72, 2008.

Onambele, G. L.; Narici, M. V. \& Maganaris, C. N. Calf muscle-tendon properties and postural balance in old age. J. Appl. Physiol., 100(6):2048-56, 2006.

Parra, J. C.; García, R. \& Santelices, I. Introducción Práctica a la Realidad Virtual. Concepción, Ediciones Universidad del Bío Bío, 2001. pp.3-4, 53-64.

Peterka, R. J. Sensorimotor integration in human postural control. J. Neurophysiol., 88(3):1097-118, 2002.

Piirtola, M. \& Era, P. Force platform measurements as predictors of falls among older people - a review. Gerontology, 52(1):1-16, 2006.
Redfern, M. S.; Yardley, L. \& Bronstein, A. M. Visual influences on balance. J. Anxiety Disord., 15(1-2):8194, 2001.

Redfern, M. S.; Jennings, J. R.; Mendelson, D. \& Nebes, R. D. Perceptual inhibition is associated with sensory integration in standing postural control among older adults. J. Gerontol. B Psychol. Sci. Soc. Sci., 64(5):569$76,2009$.

Rothermel, S.; Hale, S.; Hertel, J. \& Denegar, C. Effect of active foot positioning on the outcome of a balance training program. Phys. Ther. Sport, 5:98-103, 2004.

Seigle, B.; Ramdani, S. \& Bernard, P. L. Dynamical structure of center of pressure fluctuations in elderly people. Gait Posture, 30(2):223-6, 2009.

van Asten, W. N.; Gielen, C. C. \& Denier van der Gon, J. J. Postural adjustments induced by simulated motion of differently structured environments. Exp. Brain. Res., 73(2):371-83, 1988a.

van Asten, W. N.; Gielen, C. C.; van der Gon, J. J.Postural movements induced by rotations of visual scenes. J. Opt. Soc. Am. A, 5(10):1781-9, 1988b.

Vuillerme, N. \& Nafati, G. How attentional focus on body sway affects postural control during quiet standing. Psychol. Res., 71(2):192-200, 2007.

Wester, J. U.; Jespersen, S. M.; Nielsen, K. D. \& Neumann, L. Wobble board training after partial sprains of the lateral ligaments of the ankle: a prospective randomized study. J. Orthop. Sports Phys. Ther., 23(5):332-6, 1996.

Wolsley, C. J.; Sakellari, V. \& Bronstein, A. M. Reorientation of visually evoked postural responses by different eyein-orbit and head-on-trunk angular positions. Exp. Brain Res., 111(2):283-8, 1996.

Dirección para correspondencia:

Profesora Valeska Gatica Rojas

Escuela de Kinesiología

Departamento de Ciencias Básica

Avenida Lircay s/n

Universidad de Talca

Talca, CHILE

Email: vgatica@utalca.cl

Recibido : 20-01-2010

Aceptado: 18-02-2010 